\title{
Quantum Optics in Multiple Scattering Random Media
}

\author{
Peter Lodahl ${ }^{1,2}$ and Ad Lagendijk ${ }^{1}$
}

1 Complex Photonic Systems, Department of Science and Technology and MESA+Research Institute, Universiteit Twente, 7500 AE Enschede, The Netherlands.

2 Research Center COM, Technical University of Denmark, Dk-2800 Lyngby, Denmark.

Coherent transport of light in a disordered random medium has attracted enormous attention both from a fundamental and application point of view. Coherent wave scattering has the potential of enhancing communication capacities, is ubiquitous in acoustical and biomedical imaging, and is the basis for fundamental findings such as intensity correlations, enhanced backscattering, and Anderson localization of light. Recently, theoretical work has considered quantum optics in multiple scattering media and novel fundamental phenomena have been predicted when examining quantum fluctuations instead of merely the intensity of the light [1].

Here we present the first experimental study of the propagation of quantum noise through an elastic, multiple scattering medium [2]. Two different types of quantum noise measurements have been carried out: total transmission and short-range frequency correlations. When comparing shot noise (quantum) to technical noise (classical) we observed markedly different behavior, c.f. Fig. 1. The experimental results are found to be in excellent agreement with a quantum model for multiple scattering of light, which allows extracting both static and dynamic scattering properties of the medium. Finally, we propose a new quantum correlation in multiple scattering with no classical analog and device a way of measuring it that should be readily attainable experimentally.

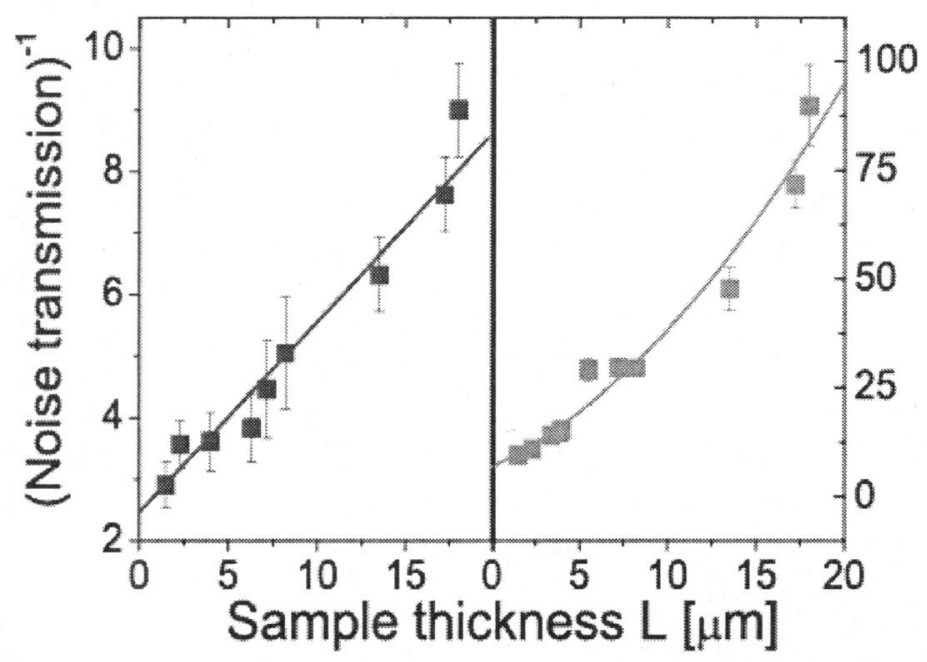

Figure 1. Inverse total transmission of shot noise (left) and technical noise (right) as a function of the thickness of the random medium. The experimental data are well explained by theory (curves).

[1] J. Tworzydlo and C.W.J. Beenakker, Phys. Rev. Lett. 89, 043902 (2002).

[2] P. Lodahl and A. Lagendijk, submitted: http://arxiv.org/abs/quant-ph/0410113. 